\title{
Dendritic cells genetically engineered to express IL-4 inhibit murine collagen-induced arthritis
}

\author{
Yoshitaka Morita, ${ }^{1}$ Jianmin Yang, ${ }^{2}$ Raj Gupta, ${ }^{1}$ Koichi Shimizu, ${ }^{3}$ Eric A. Shelden, ${ }^{4}$ \\ Judith Endres, ${ }^{1}$ James J. Mulé, ${ }^{3}$ Kevin T. McDonagh, ${ }^{2}$ and David A. Fox ${ }^{1}$ \\ ${ }^{1}$ Division of Rheumatology, Department of Internal Medicine; and Multipurpose Arthritis and \\ Musculoskeletal Diseases Center, \\ ${ }^{2}$ Division of Hematology and Oncology, Department of Internal Medicine, \\ ${ }^{3}$ Department of Surgery, and \\ ${ }^{4}$ Department of Cell and Developmental Biology, University of Michigan, Ann Arbor, Michigan, USA \\ Address correspondence to: David A. Fox, Division of Rheumatology, University of Michigan, 5520 MSRB I, \\ 1150 W. Medical Center Drive, Ann Arbor, Michigan 48109, USA. \\ Phone: (734) 647-3413; Fax: (734) 763-4151; E-mail: dfox@umich.edu.
}

Received for publication October 6, 2000, and accepted in revised form March 20, 2001.

Dendritic cells (DCs) are specialized antigen-presenting cells that migrate from the periphery to lymphoid tissues, where they activate and regulate $T$ cells. Genetic modification of DCs to express immunoregulatory molecules would provide a new immunotherapeutic strategy for autoimmune and other diseases. We have engineered bone marrow-derived DCs that express IL-4 and tested the ability of these cells to control murine collagen-induced arthritis (CIA), a model for rheumatoid arthritis in which Th1 cells play a critical role. IL-4-transduced DCs inhibited Th1 responses to collagen type II in vitro. A single injection of IL-4-transduced DCs reduced the incidence and severity of CIA and suppressed established Th1 responses and associated humoral responses, despite only transient persistence of injected DCs in the spleen. In contrast, control DCs and IL-4-transduced T cells or fibroblastic cells failed to alter the course of the disease. The functional effects correlated well with the differential efficiency of DC migration from various sites of injection to lymphoid organs, especially the spleen. The ability of splenic T cells to produce IL-4 in response to anti-CD3 was enhanced after the administration of IL-4-transduced DCs. These results support the feasibility of using genetically modified DCs for the treatment of autoimmune disease.

J. Clin. Invest. 107:1275-1284 (2001).

\section{Introduction}

Dendritic cells (DCs) are the most effective antigenpresenting cells (APCs) in the induction of primary immune responses (1). This is thought to reflect expression of high levels of MHC class II and accessory/costimulatory molecules including CD40, CD54, CD58, CD80 (B7-1), and CD86 (B7-2) by DCs. After their migration to lymphoid tissues from the periphery, DCs serve as potent APCs for T-cell activation. It was recently shown that a single injection of antigen-pulsed human DCs can lead to effective in vivo immunization (2). Recent studies have also pointed to immunoregulatory capacities of DCs (3-10). Distinct subsets of DCs have been identified in mouse (3-5), rat (6), and human (7), which preferentially induce or regulate Th1 or Th2 immune responses. A unique subpopulation of splenic DCs expressing CD8 $\alpha$ and Fas ligand may eliminate activated T cells (8). Immature DCs lacking expression of costimulatory molecules (9) or IL-10-treated DCs (10) may also possess negative immunoregulatory properties. This growing understanding of heterogeneous immunoregulatory functions of DCs prompted us to consider DC-based immunotherapies for autoimmune diseases.
Genetic modification of DCs with genes encoding immunoregulatory molecules is an attractive strategy for artificial generation of immunoregulatory DCs. This challenging approach has been tried for control of allograft rejection in transplantation. DCs genetically engineered to express vIL-10, TGF- $\beta$, or CTLA4Ig exhibit tolerogenic effects on alloreactivity (11-13). FasL-transduced DCs can prolong cardiac allograft survival in mice (14). In considering potential new immunotherapeutic strategies for autoimmune diseases, one candidate cytokine that may enhance the immunoregulatory capacities of DCs is IL-4. IL-4 is a potent mediator in shifting the balance of Th1/Th2 cells and is regarded as an anti-inflammatory cytokine. IL-4 plays its most dominant role in the differentiation of naive T cells toward a Th2 phenotype (15). IL-4 antagonizes Th1 responses by direct inhibition of IFN$\gamma$ production by activated T cells. Additionally, IL-4 reduces IL- 1 and TNF- $\alpha$ production and induces expression of the IL-1 receptor antagonist by activated macrophages. Moreover, systemic administration of IL4 has shown therapeutic potential in animal models of autoimmunity $(16-19)$. Owing to its short half-life in vivo, however, IL-4 treatment has to be given by repeated daily injection or continuous administration. Unlike 
IL-10, another important immunoregulatory cytokine, which is produced by various cell types such as monocytes/macrophages, B cells, and T cells, IL-4 is secreted by restricted cell types, mainly by $\mathrm{T}$ cells, but not by APCs. We hypothesized that DCs genetically engineered to secrete IL-4 might modify T-cell responses by migration into lymphoid tissues and direct interactions with $\mathrm{T}$ cells.

Rheumatoid arthritis (RA) is a common autoimmune disease characterized by persistent inflammation of joints resulting in progressive destruction of cartilage and bone. RA has been viewed as a primarily Th1-mediated disease (20). Addition of exogenous IL-4 to RA synovial tissue cells reduces levels of macrophage-derived proinflammatory cytokines and IFN- $\gamma(21)$. The therapeutic potential of IL-4 has also been studied in animal models of arthritis, especially collagen-induced arthritis (CIA). CIA is suppressed by IL-4 continuously administered by implanted pumps (18), or by treatment with IL-4-producing Chinese hamster ovary cells (19). More recently, adenoviral vector-mediated overexpression of IL-4 in knee joints of mice with CIA was reported to prevent cartilage destruction and bone erosion $(22,23)$. In RA, preliminary phase I trials have started using the systemic administration of IL-4 by repeated injection.

In the present study, we examined the effects of bone marrow-derived DCs genetically engineered to express IL-4 on CIA in mice. We found that a single intraperitoneal injection of a small number of IL-4-transduced DCs significantly alters the course of arthritis. The functional effects correlated well with the differential efficiency of DC migration from different sites of injection to the spleen. To our knowledge, this is the first report to evaluate immunotherapy using retrovirally transduced DCs for an autoimmune disease.

\section{Methods}

Mice. Male DBA/1 LacJ mice, 7-8 weeks old, were purchased from The Jackson Laboratory (Bar Harbor, Maine, USA). Mice were maintained in the Unit for Laboratory Animal Medicine of the University of Michigan. Animal use procedures have been approved by the University of Michigan Committee on the Use and Care of Animals.

Culture medium. Complete medium (CM) consisted of RPMI 1640 supplemented with 10\% heat-inactivated FCS, $0.1 \mathrm{mM}$ nonessential amino acids, $1 \mu \mathrm{M}$ sodium pyruvate, $2 \mathrm{mM}$ L-glutamine, $100 \mathrm{U} / \mathrm{ml}$ penicillin, 100 $\mu \mathrm{g} / \mathrm{ml}$ streptomycin, and $5 \times 10^{-5} \mathrm{M} 2-\mathrm{ME}$ (all from Life Technologies Inc., Grand Island, New York, USA). Lymphocyte medium (LM) consisted of Click's medium (Life Technologies Inc.) supplemented with $1 \%$ heatinactivated mouse serum, penicillin, streptomycin, and 2-ME. LM was used to culture spleen cells or lymph node cells from mice that had received DC injections, to avoid responses to FCS components.

Retroviral vector construction. A retroviral vector construct (pRET6) that contains long terminal repeats derived from myeloproliferative sarcoma virus as well as a puromycin resistance gene was used in the present study. cDNA encoding murine IL-4 was obtained from pNGVL3-mIL-4 (Vector Core, University of Michigan, Ann Arbor, Michigan, USA). For construction of the mIL4 retrovirus vector (pRET6-IL-4), a mIL-4 fragment of 443 bp was excised with EcoR V and Not I from pNGVL3mIL-4, and subcloned into the pBluescript vector (Stratagene, La Jolla, California, USA). A Xho I and Not I fragment from pBluescript-mIL-4 was then subcloned into pRET6. Similarly, the cDNA encoding enhanced green fluorescent protein (EGFP, obtained from pEGFP-1; CLONTECH, Palo Alto, California, USA) was subcloned into pRET6 to make pRET6-EGFP. An amphotropic Phoenix packaging cell line ( $\phi$ NXA provided by G. Nolan, Stanford University, Palo Alto, California, USA) was transiently transfected with the pRET6-mIL-4 or pRET6EGFP construct using calcium/phosphate precipitation. An ecotropic GP+E86 packaging cell line (24) was subsequently infected with filtered viral supernatants from $\phi N X A$ in the presence of protamine. After selection with puromycin $(4 \mu \mathrm{g} / \mathrm{ml})$, these polyclonal GP+E86 cells were used as producer cell lines.

Generation of retrovirally transduced DCs from bone marrow cells. Bone marrow was flushed from femurs and tibias, and depleted of erythrocytes by lysis in hypotonic buffer. Bone marrow cells $\left(10^{6} / \mathrm{ml}\right)$ were plated on $9.5 \times$ $10^{6}$ irradiated ( $30 \mathrm{~Gy}$ ) producer cells in $150 \mathrm{~cm}^{2}$ culture flasks (Becton Dickinson, Franklin Lakes, New Jersey, USA). Cells were cocultured for 2 days at $37^{\circ} \mathrm{C}, 5 \% \mathrm{CO}_{2}$ in the presence of $10 \mathrm{ng} / \mathrm{ml}$ recombinant murine GMCSF (rmGM-CSF; Immunex Corp., Seattle, Washington, USA), $10 \mathrm{ng} / \mathrm{ml}$ recombinant murine IL-4 (rmIL-4; Schering-Plough, Kenilworth, New Jersey, USA), and 8 $\mu \mathrm{g} / \mathrm{ml}$ protamine sulfate in CM. For preparing control DCs (mock-transduced DCs), bone marrow cells were cocultured with nontransduced GP+E86 cell lines. On day 2 , nonadherent cells were carefully removed from adherent producer cell lines and replated at $10^{6} / \mathrm{ml}$ in CM with rmGM-CSF and rmIL-4. On day 5, nonadherent cells were harvested and DCs were enriched by centrifugation on $14.5 \%$ metrizamide (Sigma Chemical Co., St. Louis, Missouri, USA) gradients (25). DCs were washed in HBSS before injection.

Cell surface phenotype. DCs were analyzed by direct or indirect staining with mAb's against cell surface markers. Cells were preincubated with $2.4 \mathrm{G} 2$, a rat anti-Fc receptor $\mathrm{mAb}$, and incubated with $\mathrm{PE}$-conjugated mAb's against CD11c, CD40, CD80, or CD86 (all from BD PharMingen, San Diego, California, USA). For MHC class II staining, DCs were stained with anti-I-Eq (BD PharMingen), followed by PE-conjugated antimouse IgG, $\mathrm{F}\left(\mathrm{ab}^{\prime}\right)_{2}$ (Boehringer Mannheim, Indianapolis, Indiana, USA). Flow cytometric analysis was performed using an Elite cell sorter (Coulter Electronics Ltd., Hialeah, Florida, USA).

Retroviral transduction of primary T cells and NIH 3 T3 cell line. $\mathrm{T}$ cells were enriched from splenocytes on a nylon wool column. The cells were activated for 2 days with immobilized anti-CD3 (145-2C11; BD 
PharMingen) and anti-CD28 (37.51; BD PharMingen) in CM, followed by culture for 2 days in $\mathrm{CM}$ with $300 \mathrm{IU} / \mathrm{ml}$ recombinant human IL-2 (rhIL-2; Chiron Corp., Emeryville, California, USA). For immobilized anti-CD3/anti-CD28, 24-well plates were coated overnight at $4{ }^{\circ} \mathrm{C}$ with $600 \mu \mathrm{l} /$ well of anti-CD3 and anti-CD28 $(2 \mu \mathrm{g} / \mathrm{ml}$ in PBS). Retroviral transduction was performed using filtered retroviral supernatant in 24-well plates precoated overnight at $4^{\circ} \mathrm{C}$ with 500 $\mu \mathrm{l} /$ well of RetroNectin $(80 \mu \mathrm{g} / \mathrm{ml}$ in PBS; Takara Shuzo Co., Shiga, Japan). Activated T cells, resuspended in viral supernatant at $10^{6} / \mathrm{ml}$, were added to the plates $(2 \mathrm{ml} /$ well $)$ and centrifuged at $900 \mathrm{~g}$ for 90 minutes at $32^{\circ} \mathrm{C}$. After centrifugation, the retroviral supernatants were replaced with $\mathrm{CM}$ containing rhIL-2 $(300 \mathrm{IU} / \mathrm{ml})$ and cells were incubated overnight at $37^{\circ} \mathrm{C}$. The following day, $\mathrm{T}$ cells were replated and expanded for an additional 2 days in $\mathrm{CM}$ with rhIL-2. Transduced T cells were enriched by drug selection in puromycin $(1 \mu \mathrm{g} / \mathrm{ml})$ for 3 days. Puromycin-resistant $\mathrm{T}$ cells were activated for 24 hours with immobilized anti-CD3/anti-CD28, harvested, and then washed in HBSS before injection. Murine fibroblast NIH 3T3 cells were transduced with filtered retroviral supernatant and selected with puromycin $(4 \mu \mathrm{g} / \mathrm{ml})$ for 3 days.

Induction and treatment of arthritis in mice. Mice were injected intradermally with $100 \mu \mathrm{g}$ of chicken collagen type II (CII; Chondrex, Redmond, Washington, USA) in complete adjuvant (Chondrex) at the base of the tail on day 0 . In randomized treatment groups, mice received subcutaneous, intravenous, or intraperitoneal injections of $3 \times 10^{5}$ mock-transduced, EGFP-transduced, or IL-4-transduced DCs on day 15. The subcutaneous injection was divided equally into the right and left flanks with $1.5 \times 10^{5}$ cells per site. In a separate experiment, mice received intraperitoneal injections of the indicated numbers of IL-4-transduced DCs, T cells, or NIH 3T3 cells. Limbs were individually scored every other day on a scale of $0-3$ by a blinded observer. Scores were assigned based on the amount of erythema, swelling, or joint rigidity present in each limb, giving a maximum score of 12 per mouse.

Functional DC migration assay. IL-4-transduced DCs were incubated overnight with $50 \mu \mathrm{g} / \mathrm{ml}$ of keyhole limpet hemocyanin (KLH). The KLH-pulsed DCs were washed in HBSS and injected in mice subcutaneously, intravenously, or intraperitoneally at a dose of $3 \times 10^{5}$ cells per mouse, as already described here. Spleen and inguinal lymph nodes were harvested 5 days after DC injection, and KLH-specific T-cell responses were determined by proliferation assays and IL-2 release. The isolated cells from spleen and lymph nodes were resuspended in LM. A total of $5 \times 10^{5}$ cells were cultured with or without $\mathrm{KLH}(50 \mu \mathrm{g} / \mathrm{ml})$ in 96-well flatbottom plates. Proliferation was measured in triplicate in a 72-hour assay, with $0.8 \mu \mathrm{Ci}\left[{ }^{3} \mathrm{H}\right]$ thymidine added during the last 16 hours of culture. $\left[{ }^{3} \mathrm{H}\right]$ thymidine incorporation was determined using a liquid scintillation counter. Supernatants from duplicate 48-hour cultures were stored at $-80^{\circ} \mathrm{C}$ until determination of IL-2 levels by ELISA. a
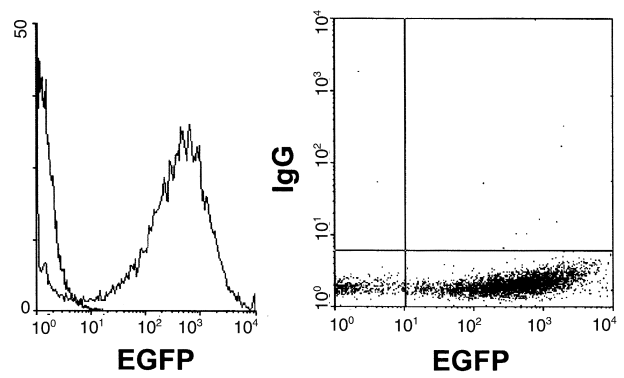

b

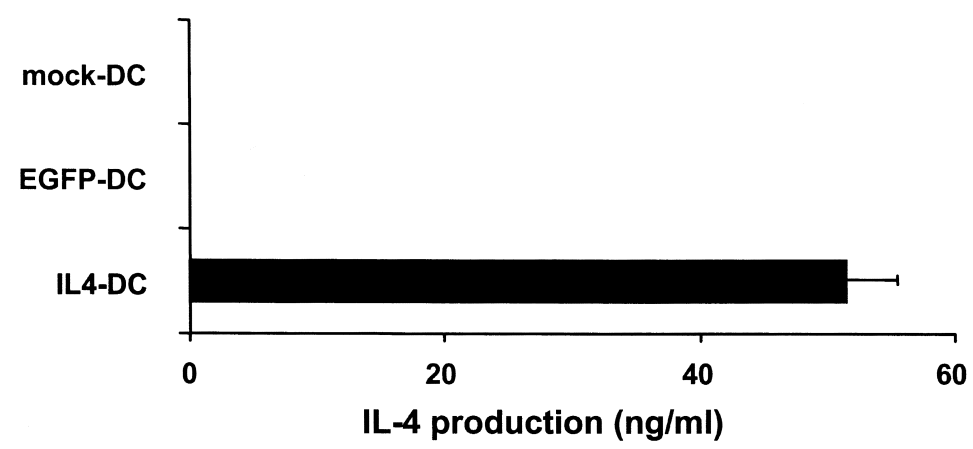

Figure 1

Expression of retrovirally transduced gene products in DCs. (a) Flow cytometric analysis of EGFP-transduced DCs. Cells were stained with PE-conjugated anti-CD11c mAb or isotype control and were analyzed for the expression of EGFP and CD11c. (b) IL-4 production by retrovirally transduced DCs. Mock-transduced, EGFP-transduced, or IL-4-transduced DCs were cultured for 24 hours at a density of $10^{6} / \mathrm{ml}$, and IL-4 levels in the supernatants were determined by ELISA. Data are the mean \pm SEM of five independent experiments. 
a

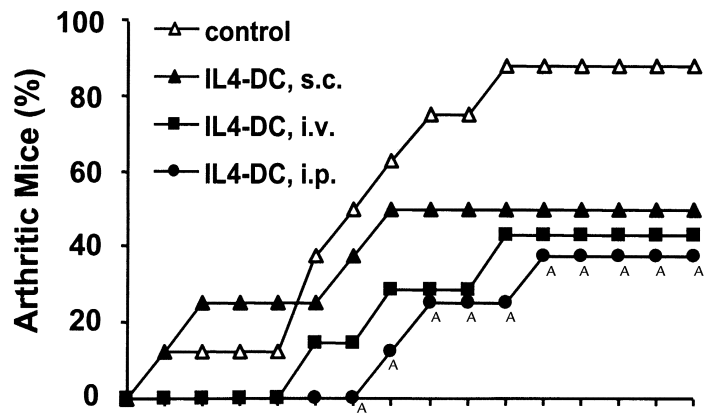

b

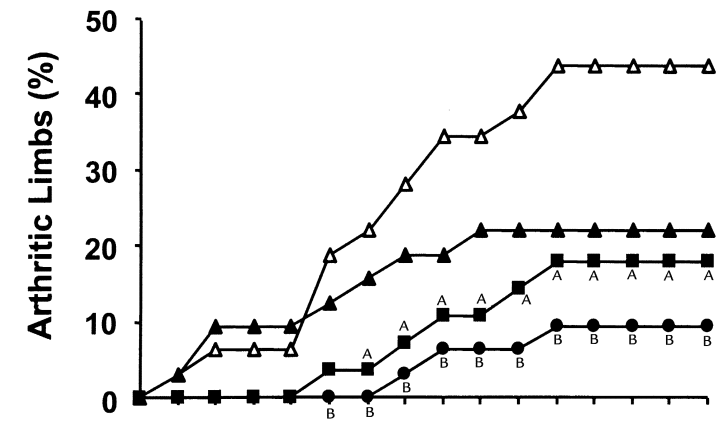

C

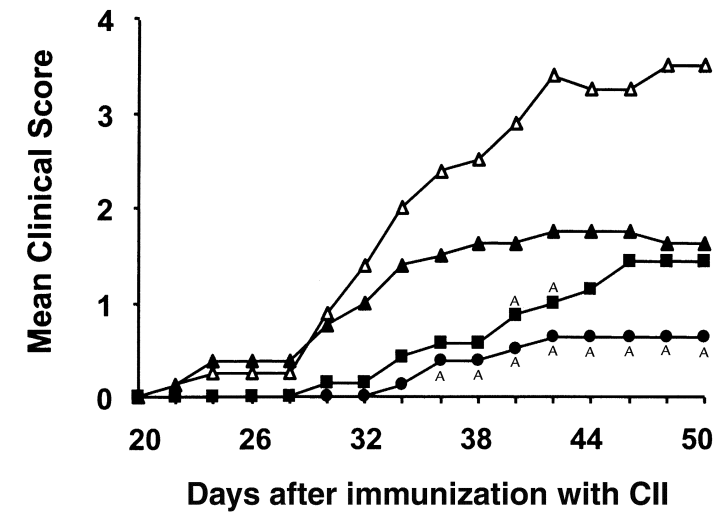

Figure 2

Effect of IL-4-transduced DCs injected subcutaneously, intravenously, or intraperitoneally on the incidence (a), mean percentages of arthritic limbs (b), and mean clinical score (c) of CIA. Mice were immunized with CII on day 0 . On day 15 , mice received subcutaneous (s.c.) $(n=$ $8)$, intravenous (i.v.) $(n=7)$, or intraperitoneal (i.p.) $(n=8)$ injections of $3 \times 10^{5}$ IL-4-transduced DC. The subcutaneous injection was performed in the right and left flanks with $1.5 \times 10^{5}$ cells in each flank. Control mice $(n=8)$ were given HBSS subcutaneously. Arthritis was monitored by a blinded observer, and clinical score was assessed as described in Methods. ${ }^{A} P<0.05$ compared with control. ${ }^{B} P<0.01$ compared with control.

Direct enumeration of in vivo migration of EGFP-transduced $D C$. A total $3 \times 10^{6}$ EGFP-transduced DCs were injected subcutaneously, intravenously, or intraperitoneally into mice. The subcutaneous. injection was performed in the right and left flanks with $1.5 \times 10^{6}$ cells, respectively. At the indicated time after injection, mice were sacrificed and spleens were harvested. The spleens were then cut into small fragments with scissors and digested with collagenase and DNase for 30 minutes at $37^{\circ} \mathrm{C}$. After depletion of erythrocytes, flow cytometric analysis was performed to detect fluorescent cells within the single-cell suspension of spleen cells. At least $2 \times 10^{6}$ living cells were analyzed. Spleen cells from untreated mice were used as a negative control. In parallel experiments, $\mathrm{EGFP}^{+}$cells within the samples were directly counted using fluorescence microscopy. By this method, cells with both green and red fluorescence were regarded as autofluorescent cells and were not counted as EGFP ${ }^{+}$cells.

Spleen cell cultures. Spleen cells were cultured in 96-well plates at a density of $5 \times 10^{6} \mathrm{cells} / \mathrm{ml}(200 \mu \mathrm{l} /$ well $)$ in LM. Cells were cultured for 72 hours with anti-CD3 (5 $\mu \mathrm{g} / \mathrm{ml}, 145-2 \mathrm{C} 11)$, or for 96 hours with medium alone or heat-denatured CII $(100 \mu \mathrm{g} / \mathrm{ml})$. Culture supernatants were stored at $-80^{\circ} \mathrm{C}$ until analyzed for cytokines by ELISA.

Cytokine assays. Murine IFN- $\gamma$, IL-2, and IL-4 were assayed by ELISA using paired antibodies (BD PharMingen) according to the manufacturer's instructions. The lower limits of detection were $40 \mathrm{pg} / \mathrm{ml}$ for IFN- $\gamma$ and IL-2, and $10 \mathrm{pg} / \mathrm{ml}$ for IL-4.

Anti-CII antibody titers. The titers of anti-CII antibodies in serum samples were determined by ELISA. All samples were measured in duplicate. Microtiter plates were coated with $5 \mu \mathrm{g} / \mathrm{ml}$ native CII (ELISA grade; Chondrex) overnight at $4{ }^{\circ} \mathrm{C}$ and then blocked by incubation with PBS containing $10 \%$ FCS. After washing with PBS $/ 0.05 \%$ Tween 20,100 $\mu$ l of diluted serum samples were added to the wells and the plates were incubated at $4^{\circ} \mathrm{C}$ overnight. Plates were washed and incubated at room temperature for 2 hours with biotinylated rat anti-mouse IgG1 or IgG2a (BD PharMingen). Plates were washed thoroughly and incubated with $2.5 \mu \mathrm{g} / \mathrm{ml}$ avidin-peroxidase (Sigma Chemical Co.) for 30 minutes. After the last wash, plates were developed with ABTS (Sigma Chemical Co.). Optical densities were determined at $405 \mathrm{~nm}$. Dilutions of a single serum sample from a CII-immunized mouse were used to generate a standard curve.

Statistical analysis. The statistical analysis was performed using $\chi^{2}$ test for values of arthritis mice and arthritis limbs or the Mann-Whitney $U$-test for clinical severity and antibody levels.

\section{Results}

Retroviral transduction of DCs to express IL-4. Retroviral delivery of genes into bone marrow-derived DCs was achieved by coculturing bone marrow cells with irradiated viral producer cell lines for 2 days, with subsequent differentiation into DCs for an additional 3 days in the presence of GM-CSF and IL-4. DCs were enriched by centrifugation on metrizamide gradients. This method consistently generated a cell population composed of more than 70\% DCs as determined by immunophenotypic analysis (MHC class II, CD80, CD86, CD11c) (data not shown). Based on the results of EGFP-transduction, more than $90 \%$ of CD $11 \mathrm{c}^{+}$DC were successfully transduced (Figure 1a). To determine the production of IL- 4 protein by the transduced DCs, supernatants of DCs cultured for an additional 24 hours after metrizamide gradient enrichment were harvested and examined for IL-4 levels using ELISA (Fig- 
a

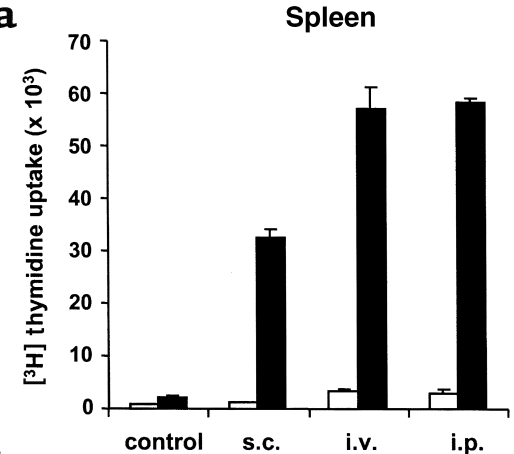

b

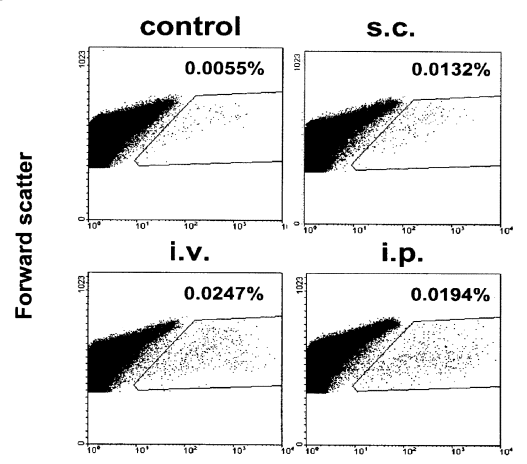

EGFP fluorescence
Lymph nodes
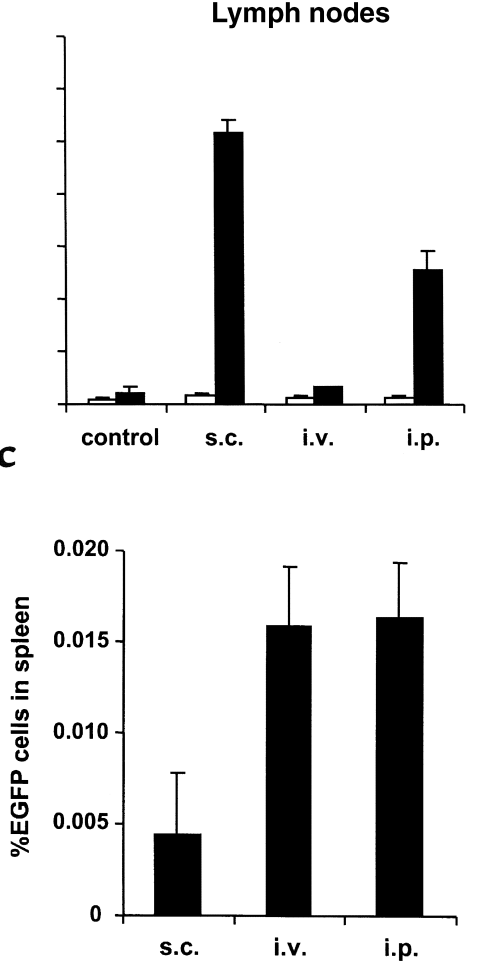

Figure 3

Efficiency of migration of DCs injected subcutaneously, intravenously, or intraperitoneally to spleen and lymph nodes. (a) Functional migration assay. A total of $3 \times 10^{5} \mathrm{IL}-4$-transduced DCs pulsed with KLH were injected subcutaneously (s.c.), intravenously (i.v.), or intraperitoneally (i.p.). Spleen and inguinal lymph nodes were harvested 5 days after DC injection, and the isolated cells were cultured with (filled bars) or without (open bars) $\mathrm{KLH}(50 \mu \mathrm{g} / \mathrm{ml})$. Cells from mice with no treatments were used as a control. Proliferation was measured as $\left[{ }^{3} \mathrm{H}\right]$ thymidine incorporation during the last 16 hours of the 72-hour culture. The results are expressed as mean cpm \pm SEM from triplicate cultures. (b and $\mathbf{c}$ ) Migration to spleen of EGFP-transduced DCs injected. A total of $3 \times 10^{6}$ EGFP-transduced DCs were injected subcutaneously, intravenously, or intraperitoneally into mice. Twenty-four hours later, spleens were removed, and the single-cell suspensions were analyzed by flow cytometry. At least $2 \times 10^{6}$ living cells were analyzed. Spleen cells from mice with no treatment were used as a negative control. Representative data are shown in $\mathbf{b}$. (c) Mean percentage \pm SEM of EGFP+ cells in spleen from two individual mice per group from which the control background is subtracted. ure $1 \mathrm{~b})$. IL-4-transduced DCs produced this cytokine at the level of $50 \mathrm{ng} / 10^{6}$ cells per 24 hours, whereas IL4 was not detected in the culture supernatants of mock-transduced or EGFP-transduced DCs.

A single injection of IL-4-transduced DCs inhibits the onset and reduces the severity of CIA. We subsequently examined the effect of IL-4-transduced DCs on CIA. In a preliminary experiment, mice received subcutaneous injections of different numbers of DCs on day 19 after immunization with CII. We found that the incidence and severity of disease development were inhibited by administration of $3 \times 10^{5}$ or $8 \times 10^{5}$ cells, but not by 1 $\times 10^{5}$ cells (data not shown). Based on this finding, $3 \times$ $10^{5}$ DCs were used in the subsequent experiments. To determine whether the effect of DCs on CIA differed according to the route of administration, mice received subcutaneous, intravenous, or intraperitoneal injections of $3 \times 10^{5}$ IL-4-transduced DCs on day 15 after CII immunization. DC injections by each route of administration inhibited the onset and reduced the severity of CIA, but the arthritis was inhibited most effectively by the intraperitoneal injection, followed by the intravenous injection, and to a lesser extent by the subcutaneous injection (Figure 2). Suppression of the development of arthritis was long-lasting for the duration of the study (50 days after immunization with CII). We next compared the effect of intraperitoneal administration of IL-4-transduced DCs with various control DCs (Table 1). In contrast to the apparent inhibitory effect of IL-4-transduced DCs on CIA, mock-transduced or EGFP-transduced DCs did not modify the course of the disease.
Inbibitory effects of IL-4-transduced DCs on CIA correlate with migration of DCs to the spleen. To examine the efficiency of migration of DCs to the spleen and lymph nodes from different sites of injections, $3 \times 10^{5}$ IL4-transduced DCs pulsed with KLH were injected subcutaneously, intravenously, or intraperitoneally. Spleen and inguinal lymph nodes were harvested 5 days after the DC injection, and the KLH-specific T-cell response was determined by proliferation assays (Figure 3a). In parallel experiments, $\left[{ }^{3} \mathrm{H}\right]$ thymidine uptake correlated well with IL-2 release in the culture (data not shown). The intraperitoneal and intravenous injections of DCs primed T cells in spleen more efficiently than did the subcutaneous injection. On the other hand, in lymph nodes the subcutaneous injection was more efficient than the intraperitoneal injection. The intravenous injection failed to consistently prime T cells in lymph nodes. The differential efficiency of T-cell priming most likely reflects the number of KLH-pulsed DCs migrating into the lymphoid organs. However, some of the splenic T-cell responses might reflect the migration of $\mathrm{T}$ cells into spleen that had been primed in lymph nodes. Therefore, DC migration into spleen was explored directly using EGFP-transduced DCs. Given that relatively small numbers of fluorescent cells were expected to be detected, higher numbers $\left(3 \times 10^{6}\right)$ of EGFP-transduced cells were injected subcutaneously, intravenously, or intraperitoneally into mice. Twentyfour hours later, spleens were removed and single-cell suspensions were analyzed to detect fluorescent cells by flow cytometry (Figure 3, b and c). Spleen cells from a mouse with no treatment were used as a negative con- 
Table 1

Effect of retrovirally transduced DCs on the incidence and severity of CIA

\begin{tabular}{lcccc}
\hline & HBSS & Mock-DC & EGFP-DC & IL4-DC \\
Arthritic mice (\%) & 87.5 & 75.0 & 62.5 & $37.5^{\mathrm{A}}$ \\
Arthritic limbs (\%) & 43.8 & 34.4 & 31.3 & $12.5^{\mathrm{B}}$ \\
Mean clinical score & $3.13 \pm 0.90$ & $2.38 \pm 0.68$ & $2.13 \pm 0.79$ & $0.88 \pm 0.52^{\mathrm{A}}$ \\
Anti-CII IgG2a (AU) & $132.0 \pm 21.2$ & $165.7 \pm 41.4$ & $142.1 \pm 30.8$ & $72.5 \pm 12.2^{\mathrm{A}}$ \\
Anti-CII lgG1 (AU) & $24.5 \pm 5.8$ & $49.7 \pm 41.4$ & $23.8 \pm 9.6$ & $27.9 \pm 9.9$
\end{tabular}

Mice were immunized with CII on day 0 . On day 15 , eight mice per group received intraperitoneal injections of $3 \times 10^{5}$ mock-transduced, EGFP-transduced, or IL-4-transduced DCs. Control animals were given HBSS. Incidence, mean percentages of arthritic limbs, mean clinical score, and serum levels of anti-CI antibodies (arbitrary units $[\mathrm{AU}]$ ) are reported at 6 weeks after immunization. Data are indicated as mean $\pm \mathrm{SEM}(n=8)$. Arthritis was monitored by a blinded observer and clinical score was assessed as described in Methods. ${ }^{A} P<0.05$ compared with control (HBSS). ${ }^{B} P<0.01$ compared with control (HBSS).

trol. Significant numbers of $\mathrm{EGFP}^{+}$cells could be detected in the spleen after the administration of the DC. Consistent with the results observed in functional assays, EGFP-transduced DCs migrated into spleen more efficiently after the intraperitoneal and intravenous injections than after the subcutaneous injection. Approximately $1 \%$ of the intraperitoneally injected EGFP-transduced DCs were detected in spleen. To confirm the quantification of $\mathrm{EGFP}^{+}$cells within the samples, $\mathrm{EGFP}^{+}$cells were also directly counted by fluorescence microscopy. $\mathrm{EGFP}^{+}$cells could be easily distinguished from autofluorescent cells and displayed a characteristic DC morphology. The superior migration of DCs after intraperitoneal and intravenous injections compared with after subcutaneous injection was confirmed by this method (data not shown).

Kinetics of the appearance of fluorescent cells in spleen was also analyzed at various times after intraperitoneal injections of EGFP-transduced DCs. These data represent not only the migration of DCs to spleen, but also the duration of transduced gene expression in the spleen. Significant numbers of EGFP ${ }^{+}$ cells could be detected at day 1 and declined slightly by day 2. The number of EGFP ${ }^{+}$cells was then decreased by day 4 (data not shown).

IL-4-transduced DCs inhibit the ongoing Th1 responses in vitro and in vivo. Both Th1 cell-mediated immunity and humoral immune responses to CII are important for onset of arthritis (26-28). To examine the ability of IL4-transduced DCs to inhibit Th1 responses to CII in vitro, spleen cells from mice that had been immunized with CII were cultured with mock-transduced or IL4-transduced DCs in the presence of CII. IL-4-transduced DCs showed significant ability to reduce IFN- $\gamma$ production in response to CII, whereas mock-transduced DCs had no effect (Figure 4). Serum levels of IgG2a and IgG1 isotypes are known to correlate with Th1 and Th2 responses, respectively, and thus can be used as an indicator of ongoing T-cell responses in vivo. Previous studies have shown the importance of IgG2a isotype of antibodies to CII to the induction of CIA. We analyzed serum levels of different isotypes of antibodies to CII after the treatment of IL-4-transduced DCs (Figure 5, Table 1). Anti-CII IgG2a levels were decreased in the groups that had been treated by IL-4-transduced
DCs, but only the intraperitoneal treatment group reached a statistically significant difference. Because anti-CII IgG1 levels varied greatly among the samples within each experimental group, no significant difference in the IgG1 levels was observed between the groups. These data suggest that IL-4-transduced DCs suppress the ongoing Th1 responses and associated antibody responses to $\mathrm{CII}$ in vivo.

Neither IL-4-transduced T cells nor IL-4-transduced NIH $3 T 3$ cells inbibit CIA. Although it seems that the unique ability of DCs to migrate into lymphoid tissues and interact directly with $\mathrm{T}$ cells is required for the suppression of CIA mediated by IL-4-transduced DCs, it might be possible to speculate that systemic levels of IL-4 secreted from these cells are enough to induce the protective effect. To address this issue further, we examined

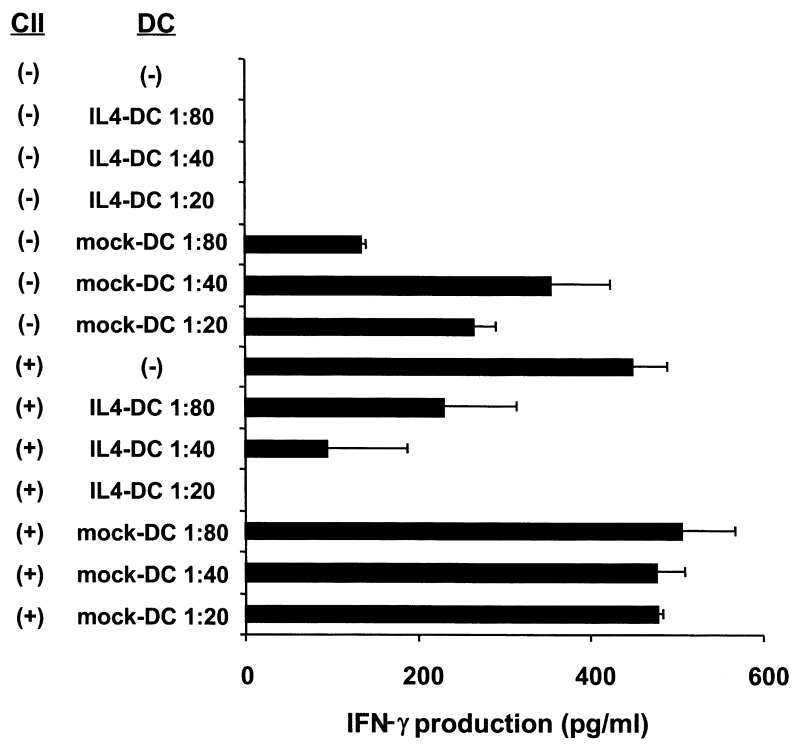

\section{Figure 4}

Effect of IL-4-transduced DCs on IFN- $\gamma$ production by spleen cells from Cll-immunized mice in vitro. Spleens were harvested from mice 15 days after immunization with CII. Cells were cultured with the indicated ratios of mock-transduced or IL-4-transduced DCs in the presence of CII $(100 \mu \mathrm{g} / \mathrm{ml})$. Ninety-six hours later, supernatants were collected and analyzed for IFN- $\gamma$ by ELISA. Data are the mean \pm SEM of duplicate cultures. 

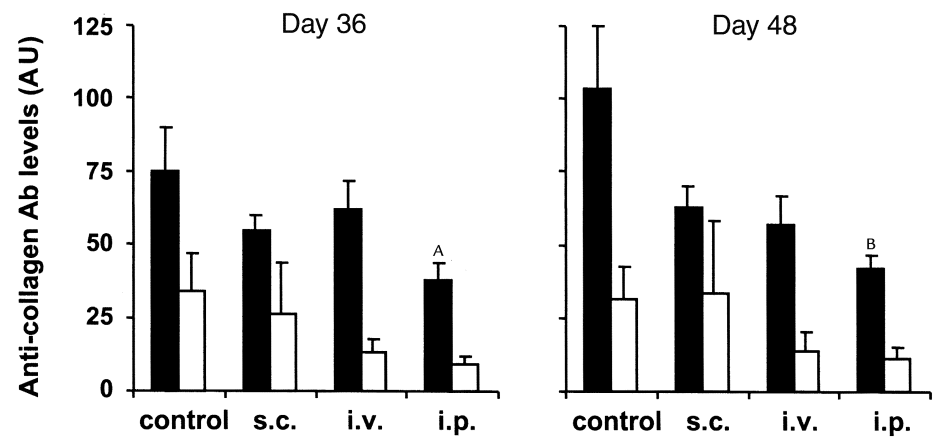

\section{Figure 5}

Effect of IL-4-transduced DCs, injected subcutaneously, intravenously, or intraperitoneally on anti-CII antibodies. Mice were immunized with $\mathrm{Cll}$ on day 0 . On day 15 , seven or eight mice per group received subcutaneous, intravenous, or intraperitoneal injections of $3 \times 10^{5} \mathrm{IL}-4$-transduced DCs. Titers of antiCII IgG2a (filled bars) and IgG1 (open bars) Ab's in sera at days 36 and 48 were measured by ELISA. Each column represents the mean arbitrary units $(A U) \pm$ SEM of seven or eight individual mice. ${ }^{A} P<0.05 \mathrm{com}-$ pared with control. ${ }^{B} P<0.01$ compared with control. whether IL-4-transduced cells other than DCs inhibit CIA. A murine fibroblast NIH 3T3 cell line transduced with IL-4 was used in this study; it was expected to produce IL-4 systemically without migration into lymphoid organs. Although these cells may be rejected by an allogeneic response, a previous study using NIH 3T3 cells transduced with IL-1Ra demonstrates that they survive and secrete the transduced product for at least 7 days in DBA/1 mice (29). IL-4-transduced NIH 3T3 cells produced this cytokine at the level of $500 \mathrm{ng} / 10^{6}$ cells per 24 hours. IL-4-transduced primary $\mathrm{T}$ cells were also tested for ability to suppress CIA. T cells may migrate into lymphoid organs, but, in contrast to DCs, they would not interact directly with other local T cells. Retroviral transduction into splenic $T$ cells activated with immobilized anti-CD3/anti-CD28 was achieved using filtered retroviral supernatant in plates precoated with RetroNectin. Approximately $50 \%$ of the $\mathrm{T}$ cells were transduced, using this protocol, as assessed by flow cytometry for EGFP-transduced $\mathrm{T}$ cells (data not shown). Transduced $\mathrm{T}$ cells were enriched by puromycin and then reactivated for 24 hours with immobilized anti-CD3/anti-CD28. Cells were washed and cultured for an additional 24 hours to measure production of IL4. These polyclonal IL-4-transduced T cells produced IL-4 at the level of $5 \mathrm{ng} / 10^{6}$ cells per 24 hours, whereas IL-4 was not detected in the culture supernatants of nontransduced T cells or EGFP-transduced T cells. The number of injected cells was adjusted based on their capacity to produce IL-4 in vitro. Therefore, mice received intraperitoneal injections of $3 \times 10^{5} \mathrm{IL}$-4-transduced DCs, $3 \times 10^{6}$ nontransduced or IL-4-transduced $\mathrm{T}$ cells, or $3 \times 10^{4}$ nontransduced or IL-4-transduced $\mathrm{NIH} 3 \mathrm{~T} 3$ cells on day 15 after CII immunization. Consistent with the results shown previously, IL-4-transduced-DC significantly altered the course of arthritis. In contrast, neither IL-4-transduced T cells nor IL4-transduced NIH 3T3 cells showed a statistically significant ability to inhibit the onset or reduce the severity of CIA (Figure 6).

IL-4-transduced DCs alter the balance of Th1 and Th2 responses in the spleen. To elucidate whether IL-4-transduced DCs could modify the T-cell cytokine pattern in vivo, spleens were removed 7 days after the intraperi- toneal administration of DCs. Spleen cells were cultured with CII or anti-CD3, and culture supernatants were then analyzed for IFN- $\gamma$ and IL-4 (Figure 7). Although IL-4 production by splenic T cells in response to CII was not detected, IL-4 production in response to anti-CD3 was substantially enhanced by the administration of IL-4-transduced DCs and was slightly enhanced by administration of mock-transduced DCs. Consistently, IFN- $\gamma$ production in response to antiCD3 was significantly decreased by administration of IL-4-transduced DCs. These results indicate that IL4-transduced DCs alter the balance of Th1/Th2 cells in the spleen and cause a shift toward a Th2 response.

\section{Discussion}

The ability of DCs to migrate to lymphoid tissues and modulate $\mathrm{T}$-cell immune responses may provide a new strategy for treatment of autoimmune diseases. The experiments described here support the feasibility of using genetically modified DCs for this purpose. The duration of expression of the virally encoded gene in spleen appears to be very limited based on the kinetics of the appearance of $\mathrm{EGFP}^{+}$cells. These results indicate the number of EGFP ${ }^{+}$cells was decreased by day 4 after administration. Although this finding could be explained by several possible scenarios, a likely explanation is that the DCs were eliminated by apoptosis during the interaction with activated T cells $(30,31)$. Importantly, despite transient persistence of injected DCs in the spleen, IL-4-transduced DCs inhibited pathogenic Th1 responses in vivo. It is reported that continuous administration of IL-4 by implanted pump delays the onset of CIA and that the suppression is associated with decreased levels of anti-CII IgG2a (18). However, it should be emphasized that in our study, the ongoing Th1 response was suppressed by a single injection of IL-4-transduced DCs.

Several experimental approaches support the hypothesis that the inhibitory effect of IL-4-transduced DCs on CIA is due to their migration into the lymphoid organs in which pathogenic immune reactions occur. First, mice that received IL-4-transduced DCs were bled at days 1,4 , or 7 , and serum levels of IL-4 were determined by ELISA. No cytokine was detected in the 

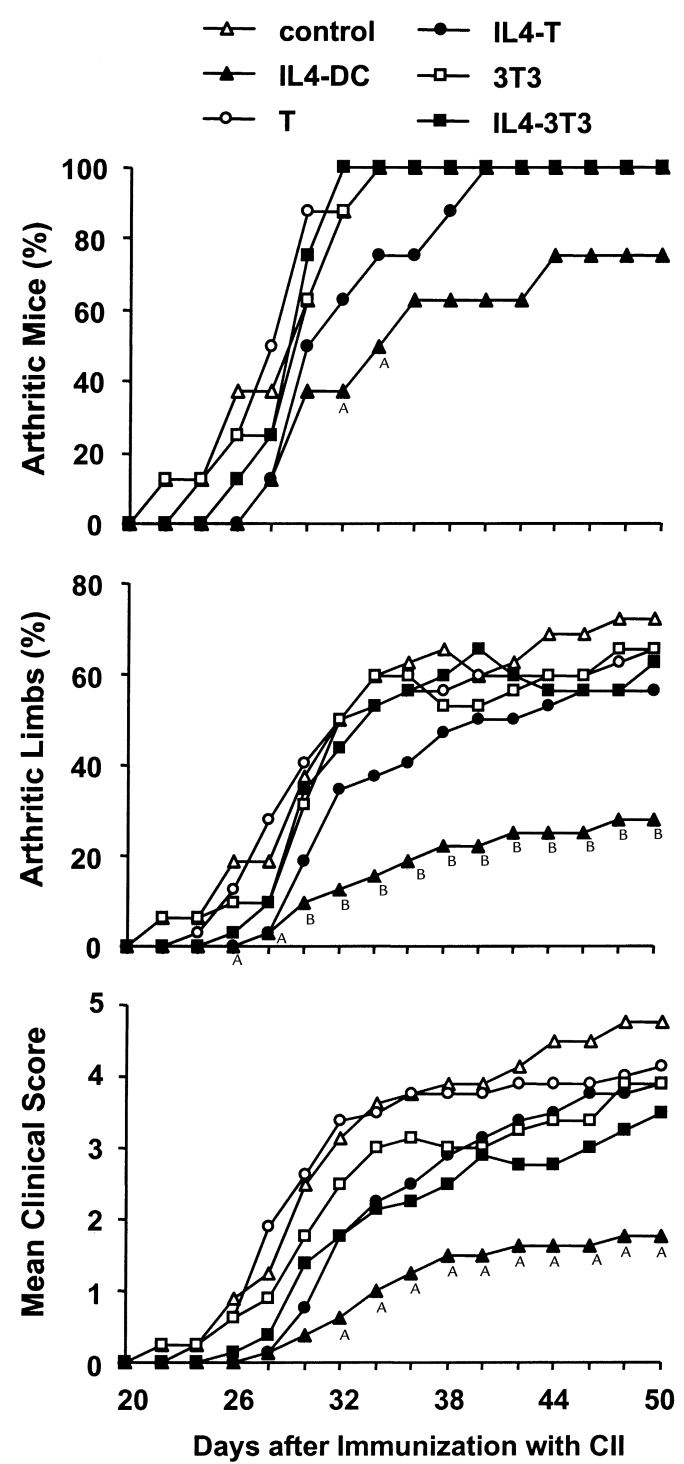

serum, even after 10 times higher numbers of IL4 -transduced DCs $\left(3 \times 10^{6}\right)$ were administered. This finding suggests that it is unlikely that disease suppression was due to high systemic levels of IL-4. Second, the effects of DCs differed according to the route of administration; the arthritis was inhibited most effectively by the intraperitoneal injection, followed by the intravenous injection, and to a lesser extent by the subcutaneous injection. The analysis of DC migration indicates that the inhibitory effect of IL-4-transduced DCs on CIA correlates with migration of DCs to the

\section{Figure 7}

Effect of IL-4-transduced DCs on T-cell cytokine pattern in spleen. Mice were immunized with $\mathrm{Cll}$ on day 0 . On day 15 , mice received intraperitoneal injections of $3 \times 10^{5}$ mock-transduced (gray bars) or IL-4-transduced (filled bars) DCs. Control animals were given HBSS (open bars). On day 21 , spleens were harvested. Cells were cultured for 72 hours with anti-CD3 $(5 \mu \mathrm{g} / \mathrm{ml})$ or for 96 hours in the presence of CII $(100 \mu \mathrm{g} / \mathrm{ml})$. Culture supernatants were analyzed for IFN- $\gamma$ and IL- 4 by ELISA. Data are the mean \pm SEM of triplicate cultures. The data shown are from a single experiment representative of two identical experiments. lymphoid organs. Considering that the intravenous injection is more effective than the subcutaneous injection, migration of DCs into the spleen may be more important for the treatment of this model than is their migration into inguinal lymph nodes. This conclusion is also consistent with data showing that the intraperitoneal injection is the most effective, as DCs could migrate efficiently into the spleen (as well as inguinal lymph nodes) by this route. Third, CIA was not suppressed by IL-4-transduced T cells or by the IL-4-transduced fibroblast cell line. It is especially instructive that IL-4-transduced polyclonal T cells did not inhibit CIA, despite their potential to migrate into the spleen. On the basis of these results, we conclude that the ability of IL-4-transduced DCs to modulate CIA does not result from simple delivery of IL-4 to the lymphoid organs, but may also require specific interactions between splenic T cells and IL-4 secreting DCs.

We propose that the effect of IL-4-transduced DC migration into lymphoid organs is to generate an altered balance of Th1/Th2 cells in the spleen and a shift toward a Th2 response. The ability of splenic $\mathrm{T}$ cells to produce IL-4 in response to anti-CD3 was enhanced after the administration of IL-4-transduced

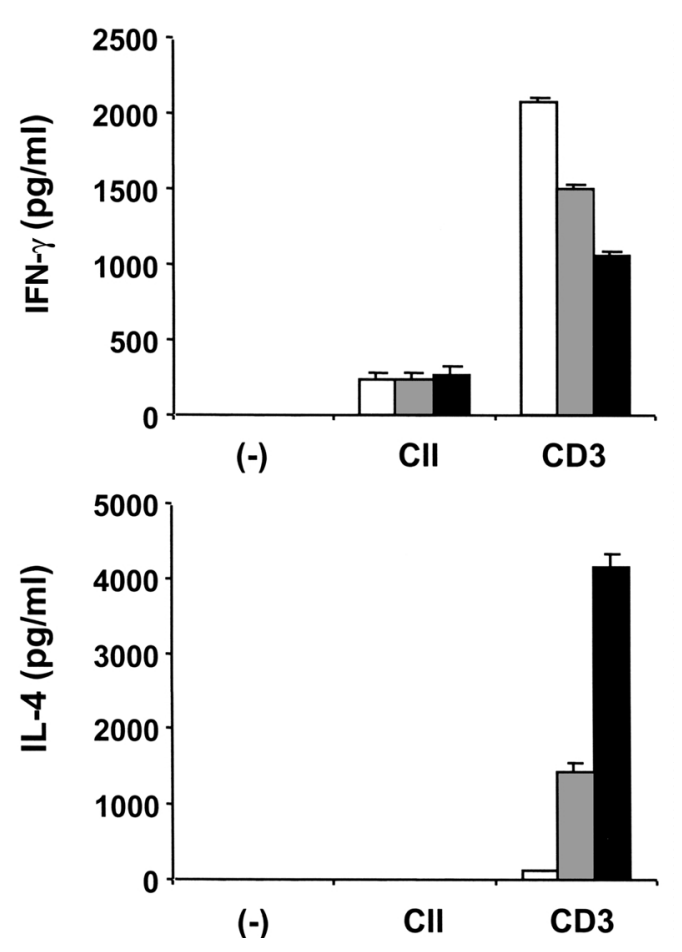


DCs. Therefore, we speculate that such Th2 cells within lymphoid organs inhibit the neighboring pathogenic Th1 response in the microenvironment and contribute to long-lasting suppression of the arthritogenic Th1 immune response in vivo. Such effects can occur despite the continued presence of potentially pathogenic Th1 cells in lymphoid organs, as demonstrated by the preserved ability of splenocytes to produce IFN$\gamma$ ex vivo when challenged with CII (Figure 7).

Genetically modified pathogenic $\mathrm{T}$ cells that express IL-4 or IL-10 have been shown to ameliorate experimental autoimmune encephalomyelitis (EAE), another Th1-mediated disease $(32,33)$. Although both T cells and DCs have the ability to migrate to lymphoid organs or sites of disease, their mechanisms seem to be quite different. Our strategy was designed to modify local T-cell responses by transient introduction of IL4-transduced DCs, whereas strategies that have used genetically modified pathogenic $T$ cells are designed to express the transduced IL- 4 or IL-10 gene for a long time at sites of disease. The transient expression of a transduced gene in vivo by DCs suggests that this approach may be a safer and more physiological means to modulate autoimmune disease. A logical concern is that DCs might augment the pathogenic immune response under some conditions. However, in our study, the severity of disease was not enhanced even by administration of control DCs.

IL-4 is only one of several genes that appear to have potential for the treatment of autoimmune diseases. Soluble IL-15 receptor, for example, was reported to prevent CIA (34). To test its therapeutic ability, we have generated soluble IL-15 receptor-transduced DCs, but no significant effect on CIA was observed after a single injection of these cells, given at day 15 after immunization with CII (Y. Morita and D.A. Fox, unpublished data). These results suggest that cytokine inhibitor genes, which may require long-term expression in vivo to obtain therapeutic effects, might not be optimal candidates for treatment of autoimmune diseases with single injections of transduced DCs.

The present studies demonstrate therapeutic efficacy of IL-4-transduced DCs for prophylactic treatment of an autoimmune disease. Further studies, perhaps using more chronic inflammatory model systems, would be required before considering their therapeutic application in chronic human diseases such as RA. This may involve additional modification of IL-4-transduced DCs to enhance their immunoregulatory capacities. Injection of a nonpathogenic antigen into mice after immunization with IL-4-transduced DCs pulsed with the same antigen could potentially cause production of Th 2 cytokines in vivo, which in turn might suppress a pathogenic Th1 response more strongly. This therapeutic approach, known as bystander suppression, has proved to be effective in EAE, in which immunization with KLH was used to induce a nonpathogenic Th2 response (35) and regulatory IL-10 production (36) that overcame a pathogenic Th1 response. Con- sidering the fact that the pathogenic antigen in RA is unknown (37), and considering the obvious risks for utilizing such an antigen for immunotherapy even if it is identified, this approach using a nonpathogenic antigen may be more realistic for human application, compared with use of genetically modified DCs pulsed with putative RA autoantigens.

In conclusion, this report demonstrates a therapeutic effect of genetically modified DCs in an experimental autoimmune disease. Our findings suggest that DCs modulated the autoimmune response in vivo not only by delivery of the transduced immunoregulatory gene product, but also by specific interactions with $\mathrm{T}$ cells in lymphoid tissues. With further understanding of regulatory functions of DCs and evaluation of alternative approaches to modification of DCs, such as transduction of other immunoregulatory genes or use of antigens, genetically modified DCs might become a potent therapeutic option for the treatment of autoimmune disease.

Note added in proof. Very recently, Kim and colleagues have suppressed CIA in mice by using intravenous injection of DCs infected with an adenoviral vector that expressed IL-4. (Kim, S.H., et al. 2001. Effective treatment of established murine collagen-induced arthritis by systemic administration of dendritic cells genetically modified to express IL-4. J. Immunol. 166:3499-3505.)

\section{Acknowledgments}

We thank E. Thomas of Immunex Corp. and S. Narula of Schering-Plough for providing rmGM-CSF and rmIL-4, respectively; G. Nolan for the Phoenix packaging cell line; B.J. Roessler and L.J. Crofford for critical reading of the manuscript; and L.K. Myers, E. Parks, and J. MacGregor for helpful advice. This work was supported by a research grant from the Arthritis Foundation (to Y. Morita), by grants from the NIH (DK02349 to K.T. McDonagh and AR38477 to D.A. Fox), and by the University of Michigan Multipurpose Arthritis and Musculoskeletal Diseases Center.

1. Banchereau, J., and Steinman, R.M. 1998. Dendritic cells and the control of immunity. Nature. 392:245-252.

2. Dhodapkar, M.V., et al. 1999. Rapid generation of broad T-cell immunity in humans after a single injection of mature dendritic cells. J. Clin. Invest. 104:173-180.

3. Pulendran, B., et al. 1999. Distinct dendritic cell subsets differentially regulate the class of immune responses in vivo. Proc. Natl. Acad. Sci. USA. 96:1036-1041.

4. Maldonado-López, R., et al. 1999. CD8 $\alpha^{+}$and CD8 $\alpha^{-}$subclasses of dendritic cells direct the development of distinct T helper cells in vivo. J. Exp. Med. 189:587-592.

5. Iwasaki, A., and Kelsall, B.L. 1999. Freshly isolated Peyer's patch, but not spleen, dendritic cells produce interleukin 10 and induce the differentiation of T helper type 2 cells. J. Exp. Med. 190:229-239.

6. Stumbles, P.A., et al. 1998. Resting respiratory tract dendritic cells preferentially stimulate $T$ helper cell type 2 (Th2) responses and require obligatory cytokine signals for induction of Th1 immunity. J. Exp. Med. 188:2019-2031.

7. Rissoan, M.-C., et al. 1999. Reciprocal control of T helper cell and dendritic cell differentiation. Science. 283:1183-1186.

8. Süss, G., and Shortman, K. 1996. A subclass of dendritic cells kills CD4 T cells via Fas/Fas-ligand-induced apoptosis. J. Exp. Med. 183:1789-1796.

9. Lu, L., McCaslin, D., Starzl, T.E., and Thomson, A.W. 1995. Bone marrow-derived dendritic cell progenitors (NLDC $145^{+}$, MHC class $\mathrm{II}^{+}, \mathrm{B} 7-$ $\left.1^{\mathrm{dim}}, \mathrm{B} 7-2^{-}\right)$induce alloantigen-specific hyporesponsiveness in murine $\mathrm{T}$ 
lymphocytes. Transplantation. 60:1539-1545.

10. Steinbrink, K., Wölfl, M., Jonuleit, H., Knop, J., and Enk, A.H. 1997. Induction of tolerance by IL-10-treated dendritic cells. J. Immunol. 159:4772-4780.

11. Takayama, T., et al. 1998. Retroviral delivery of viral interleukin-10 into myeloid dendritic cells markedly inhibits their allostimulatory activity and promotes the induction of T-cell hyporesponsiveness. Transplantation. 66:1567-1574.

12. Lee, W.-C., et al. 1998. Phenotype, function, and in vitro migration and survival of allogeneic dendritic cell progenitors genetically engineered to express TGF- $\beta$. Transplantation. 66:1810-1817.

13. Lu, L., et al. 1999. Adenoviral delivery of CTLA4Ig into myeloid dendritic cells promotes their in vitro tolerogenicity and survival in allogeneic recipients. Gene Ther. 6:554-563.

14. Min, W.-P., et al. 2000. Dendritic cells genetically engineered to express Fas ligand induce donor-specific hyporesponsiveness and prolong allograft survival. J. Immunol. 164:161-167.

15. O'Garra, A. 1998. Cytokines induce the development of functionally heterogeneous T helper cell subsets. Immunity. 8:275-283.

16. Racke, M.K., et al. 1994. Cytokine-induced immune deviation as a therapy for inflammatory autoimmune disease. J. Exp. Med. 180:1961-1966.

17. Rapoport, M.J., et al. 1993. Interleukin 4 reverses T cell proliferative unresponsiveness and prevents the onset of diabetes in nonobese diabetic mice. J. Exp. Med. 178:87-99.

18. Horsfall, A.C., et al. 1997. Suppression of collagen-induced arthritis by continuous administration of IL-4. J. Immunol. 159:5687-5696.

19. Bessis, N., et al. 1996. Attenuation of collagen-induced arthritis in mice by treatment with vector cells engineered to secrete interleukin-13. Eur. J. Immunol. 26:2399-2403.

20. Miossec, P., and van den Berg, W. 1997. Th1/Th2 cytokine balance in arthritis. Arthritis Rheum. 40:2105-2115.

21. Miossec, P., et al. 1992. Inhibition of the production of proinflammatory cytokines and immunoglobulins by interleukin- 4 in an ex vivo model of rheumatoid synovitis. Arthritis Rheum. 35:874-883.

22. Lubberts, E., et al. 1999. Adenoviral vector-mediated overexpression of IL-4 in the knee joint of mice with collagen-induced arthritis prevents cartilage destruction. J. Immunol. 163:4546-4556.

23. Lubberts, E., et al. 2000. IL-4 gene therapy for collagen arthritis suppresses synovial IL-17 and osteoprotegerin ligand and prevents bone erosion. J. Clin. Invest. 105:1697-1710.

24. Markowitz, D., Goff, S., and Bank, A. 1988. A safe packaging line for gene transfer: separating viral genes on two different plasmids. J. Virol.
62:1120-1124.

25. Fields, R.C., Shimizu, K., and Mulé, J.J. 1998. Murine dendritic cells pulsed with whole tumor lysates mediate potent antitumor immune responses in vitro and in vivo. Proc. Natl. Acad. Sci. USA. 95:9482-9487.

26. Seki, N., et al. 1988. Type II collagen-induced murine arthritis. I. Induction and perpetuation of arthritis require synergy between humoral and cell-mediated immunity. J. Immunol. 140:1477-1484.

27. Mauri, C., Williams, R.O., Walmsley, M., and Feldmann, M. 1996. Relationship between Th1/Th2 cytokine patterns and the arthritogenic response in collagen-induced arthritis. Eur. J. Immunol. 26:1511-1518.

28. Chu, C.-Q., and Londei, M. 1996. Induction of Th2 cytokines and control of collagen-induced arthritis by nondepleting anti-CD4 Abs. J. Immunol. 157:2685-2689.

29. Bakker, A.C., et al. 1997. Prevention of murine collagen-induced arthritis in the knee and ipsilateral paw by local expression of human interleukin-1 receptor antagonist protein in the knee. Arthritis Rheum. 40:893-900.

30. Ingulli, E., Mondino, A., Khoruts, A., and Jenkins, M.K. 1997. In vivo detection of dendritic cell antigen presentation to $\mathrm{CD}^{+} \mathrm{T}$ cells. J. Exp. Med. 185:2133-2141.

31. Matsue, H., et al. 1999. Dendritic cells undergo rapid apoptosis in vitro during antigen-specific interaction with $\mathrm{CD}^{+} \mathrm{T}$ cells. J. Immunol. 162:5287-5298.

32. Shaw, M.K., et al. 1997. Local delivery of interleukin 4 by retrovirus-transduced $\mathrm{T}$ lymphocytes ameliorates experimental autoimmune encephalomyelitis. J. Exp. Med. 185:1711-1714.

33. Mathisen, P.M., Yu, M., Johnson, J.M., Drazba, J.A., and Tuohy, V.K. 1997. Treatment of experimental autoimmune encephalomyelitis with genetically modified memory T cells. J. Exp. Med. 186:159-164.

34. Ruchatz, H., Leung, B.P., Wei, X.-Q., McInnes, I.B., and Liew, F.Y. 1998. Soluble IL-15 receptor $\alpha$-chain administration prevents murine collagen-induced arthritis: a role for IL-15 in development of antigeninduced immunopathology. J. Immunol. 160:5654-5660.

35. Falcone, M., and Bloom, B.R. 1997. A T helper cell 2 (Th2) immune response against non-self antigens modifies the cytokine profile of autoimmune $\mathrm{T}$ cells and protects against experimental allergic encephalomyelitis. J. Exp. Med. 185:901-907.

36. Stohlman, S.A., Pei, L., Cua, D.J., Li, Z., and Hinton, D.R. 1999. Activation of regulatory cells suppresses experimental allergic encephalomyelitis via secretion of IL-10. J. Immunol. 163:6338-6344.

37. Fox, D.A. 1997. The role of $\mathrm{T}$ cells in the immunopathogenesis of rheumatoid arthritis: new perspectives. Arthritis Rheum. 40:598-609. 